\title{
A Scalable Code for Reducing Quantum Errors
}

\section{A new scheme could offer a technologically viable solution for remedying computational errors in near-term quantum devices.}

\author{
By Sophia Chen
}

f quantum computers are to be useful in the near-term, they will require methods for remedying computational mistakes that arise from imperfect hardware. However, most error correction schemes are too computationally costly to implement on existing machines, as they require encoding a single bit of quantum information into thousands of physical qubits. The complexity of these schemes is prohibitive for existing and near-term quantum computers. In new work, Bálint Koczor of the University of Oxford, UK, presents a method for side-stepping error correction by reducing errors in the first place [1]. The new method should offer better scaling to larger devices than existing error correction codes.

The algorithm suppresses errors for a fundamental operation that is the basis of promising quantum computing applications such as the calculation of molecular ground states: estimating the expectation value of an observable. The new scheme involves performing the same operations in parallel on multiple sets of qubits, where each qubit is prepared in the same quantum state. If all the qubits were identical and error-free,

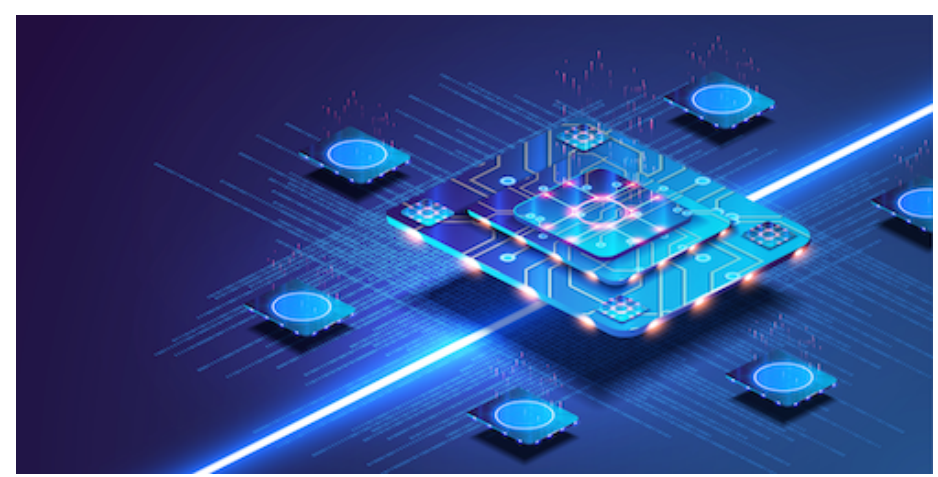

Credit: ZinetroN/stock.adobe.com swapping them would not affect the outcome of the calculation. By swapping the order of the qubit interactions in multiple copies of the circuit, the scheme can identify the circuit copies that have errors and filter out their contributions.

Simulating a specific quantum circuit consisting of several hundred quantum gates, Koczor finds that the scheme achieves an error rate sufficient for many applications on existing noisy quantum computers at the expense of adding no more than four copies of the circuit. With an independent team involving Google researchers recently proposing a similar scheme, qubit-swapping approaches are gaining momentum as error-reduction solutions for near-term quantum computers, says Koczor.

Sophia Chen is a freelance science writer based in Columbus, Ohio.

\section{REFERENCES}

1. B. Koczor, "Exponential error suppression for near-term quantum devices," Phys. Rev. X 11, 031057 (2021). 